\title{
Transdisciplinary Features of Cognitive Semiotics
}

\author{
Zhang Lihong \\ School of Foreign Language Education, Liaocheng University, Liaocheng, 252059
}

Keywords: cognitive semiotics, Transdisciplinarity, Ideology, Cognitive poetics

\begin{abstract}
Transdisciplinarity is a new orientation of interdisciplinary research. Cognitive semiotics is often regarded as the interdisciplinary and cross-disciplinary studies of languages and symbols using many theories and methods of related subjects such as the cognitive science, semiotics, aesthetic, linguistics, psychology and other disciplines. From the perspective of transdisciplinarity and the theoretical sources, cognitive semiotics is analyzed, providing a direction for the development of linguistics and semiotics.
\end{abstract}

\section{Introduction}

The concept of transdisciplinarity was first proposed by Piaget in 1972. Gibbons et al. (1994) argue that research must break through the original disciplines of theoretical concepts and patterns, and reconstruct relevant cognitive and social practices, in stead of simple use of the theory and method of existing discipline. Hu Zhuang-lin (2012:21) thinks transdisciplinarity aims to understand the current world across subjects and others. He thinks transdisciplinarity includes five aspects: the second mode of knowledge production, humanistic (the interaction between the subject and object), the unity of the knowledge, the different levels of reality, and three axioms. Li Ying (2013) and Zhou Pin(2014), based on the concept of transdisciplinarity, elaborated the reason of the English teaching mode, as well as curriculum system reform of linguistics. Jjiang Yi-min (2009) and Wang Yong(2014) discussed production mode of transdisciplinarity. From the above, although study of transdisciplinarity is not much, but whether it is in the field of natural science, social science or humanities, the study of transdisciplinarity can help us find, observe, analyze and solve problems fully and completely.

\section{The cognitive semiotics and related research}

In 1995, Daddesio's Mind and Symbol: the Correlation of Cognitive Science and Semiology first proposed the concept of Cognitive Semiotics. In 2007 the journal of cognitive semiotics officially published in Denmark marked the gradual maturity of cognitive semiotics. Cognitive semiotics studies cultural symbol from the aspects of cognitive study of language, and explores the understanding of human meaning generation. Its ultimate goal is to integrate the vocabulary and sentence level to fully understand the significance of discourse. As a new member in the field of cognitive semiotics, it is between different disciplines, even beyond other disciplines..(Liu Li, 2013:22) Cognitive semiotics can provide a new view of the world, combining biology, phenomenology and thinking science, which is the essence of transdisciplinarity.(Hu Zhuang-lin, 2012:21)

Cognitive semiotics starts late in our country. Through accurate input "cognitive semiotics" in CNKI, 13 articles can be searched out, including Hu Zhuang-lin "cognitive semiotics" (2010) and “The development of cognitive semiotics research in China” (2013)etc. Su Xiao-jun (2007) is the 
earliest researcher of cognitive semiotics. Hu Zhuang-lin not only analyzes the six main content of cognitive semiotics, that is agency, consciousness, cognitive poetics, new structure, human semiotics and biological semiotics, aesthetics, and discusses the theoretical discussion and practical application of cognitive linguistics various experts and scholars posed. Liu Li (2013) analyzed the origin of cognitive semiotics, and the possibility of the development of cognitive semiotics from the cognitive rules of symbolic representation, cognitive representation and social cognitive theory. Wang Yin(2011:4) proposed that cognitive semiotics is closely related to cognitive linguistics. The former benefits from research achievements of the latter, and both have a common core principles, namely the principle of "realistic--cognitive-language". Guo Hong (2005) by comparing the basic theory of cognitive semiotics and cognitive linguistics, found they have the consistency in the philosophical basis, method and application scope. Cognitive semiotics has obtained a degree of attention, but there is no more research about the transdisciplinarity of cognitive semiotics

\section{Transdisciplinarity of cognitive semiotics}

Cognitive semiotics is a strong and cross edge discipline, which covers semiotics, linguistics, cognitive science and psychology theory, and it has penetrated into economics, sociology, anthropology, culture and other fields of science and social science. Its related theory has revealed the unique charming in linguistics, literary criticism, and architectural theory. Cognitive semiotics is considered to be an interdisciplinary, interdisciplinary methodology. The interdisciplinary of cognitive semiotics decomposed the fixed object or natural object from the original science into a new subject, thus appeared some new objects belong to different disciplines. These new objects associated with each other in its natural form, thus forming a combination of natural object in the various disciplines. This combination can be called transdisciplinarity.

As the combination of cognitive science and semiotics, the position of cognitive semiotics has not yet formed a unified thought. In order to understand the subject position of cognitive semiotics, the position of semiotics and cognitive science have to be talked about. Semiotics appeared at the beginning of the 20th century, is a systematic study of language symbols and non-linguistic symbols. The Swiss linguist Saussure and American logician Pierce were the founder of modern semiotics. Semiotics is the combination of theory and the connotation of many subjects, whose methodology has penetrated science and social science fields, promoting the interdisciplinary communication between each other, which breaks the "barriers" to other disciplines. Cognitive science is the contemporary emerging category, trying to explore the nature, constitution, development and application of knowledge. It is considered as the integration of spsychology, linguistics, neurophysiology, of computer science and other disciplines. Cognitive semiotics should not be seen as a simple addition of cognitive science and semiotics. Through the observation of cognitive science and the differences and similarities between the two disciplines, we can discover cognitive science emphasizes the brain cognitive processing of the world. it relies on test and computer simulation, etc., while the semiotics main focuses on discourse analysis and theory construction on the basis of the concepts such as natural science, social science and symbols.

The author thinks that going beyond the two "independent discipline” in a larger extent can highlight the advantages of multidisciplinary, thus to relate the two together. The transdisciplinarity of cognitive semiotics is self-evident.

Modern linguistics is the basis and the main source of cognitive semiotics. Both have special close contact. Saussure thought, modern structuralist linguistics and cognitive semiotics are overlapped each other on the content, theory and applications. Thanks to the autonomy and independence of modern linguistics, cognitive semiotics shows a relatively clear outline. If not on 
the basis of modern linguistics, clarity or suitability of will be weaker.

Both modern linguistics, humanities and social science, natural science and the modern philosophy have more or less influence on cognitive semiotics. But one thing is consistent: aparting from any field, cognitive semiotics research will not achieve effective results. It is beyond all the inherent range of related disciplines, including the materials, methods, style and the evaluation criteria. Therefore, we need to break through the framework of mainstream discipline to play to the unique creativity and imagination. In this sense, transdisciplinarity in cognitive semiotics research will occupy the central position.

\section{Conclusion}

To sum up, characteristics of the cognitive semiotics and ideological source reflect the characteristics of transdisciplinarity. Cognitive linguistics will continue along the way of study of transdisciplinarity, and breakthrough the control of the boundaries of subject in order to solve all the problems with all my heart.

\section{References}

[1] Hu Jun. The Research Progress and Its Prospect of Cognitive Aesthetic in Contemporary China [J]. Journal of social science, 2014, (4) : 184-192.

[2] Hu Zhuang-lin. Cognitive semiotics [J]. Journal of foreign languages, 2010, (5) : 20 - 25.

[3] Jiang Yi-min. Transdisciplinary As a New Form of Research [J]. Zhejiang Social Sciences, 2009, (1) : 8-16.

[4] Li Ying. A Transdisciplinary Study on the Motives of Teaching with English as a

Medium of Instruction[J]. Chinese Foreign Language, 2013, (1) : 47-53.

[5] Liu Li. Cognitive semiotics and Its Development [J]. Journal of Lanzhou University (Social Sciences), 2013, (2) : 22-26.

[6]Su Xiao-jun. A Cognitive Semiotics of Easter Wings[J]. Foreign Language Research, 2007, (1) : 121-124. 